\title{
The Study of GPX3 Methylation in Patients with Kashin-Beck Disease and its mechanism in chondrocyte apoptosis
}

Lixin $\mathrm{Han}^{1 \S}$, Xiaoli Yang ${ }^{1 \S}$, Wenyan $\mathrm{Sun}^{1}$, Zhaofang $\mathrm{Li}^{1}$, Hao Ren ${ }^{1}$, Baorong $\mathrm{Li}^{1}$, Rongqiang Zhang ${ }^{1}$, Dandan Zhang ${ }^{1}$, Ziyun $\mathrm{Shi}^{1}$, Jifeng Liu ${ }^{1}$, Junling Cao ${ }^{1}$, Jianjun Zhang ${ }^{2}$, Yongmin Xiong $^{1 *}$

1. Institute of Endemic Diseases and Key Laboratory of Trace Elements and Endemic

Diseases, National Health Commission of the People's Republic of China, School of Public Health, Xi'an Jiaotong University Health Science Center, No.76 Yanta West Road, Xi'an, Shaanxi 710061, P.R. China.

2. Department of Epidemiology, Indiana University Richard M. Fairbanks School of Public Health, Indianapolis, 1050 Wishard Boulevard, IN 46202, USA

$\S$ Co-first author

*Corresponding author: Yongmin Xiong. Institute of Endemic Diseases, School of Public Health, Xi'an Jiaotong University Health Science Center, No 76 Yanta West Road, Xi'an, Shaanxi 710061, P. R. China. Tel: 86-29-82655193, Fax: +86-29-82655032, Email address: xiongym@mail.xjtu.edu.cn.

Shortened version of the title: GPX3 methylation in Kashin-Beck Disease

Grant Support: This study was supported by grants from National Natural Science Foundation of China (No. 81573104, 81773372, 81172610 and 81673117).

This is the author's manuscript of the article published in final edited form as:

Han, L., Yang, X., Sun, W., Li, Z., Ren, H., Li, B., ... \& Cao, J. (2018). The study of GPX3 methylation in patients with Kashin-Beck Disease and its mechanism in chondrocyte apoptosis. Bone, 117, 15-22. https://doi.org/10.1016/j.bone.2018.08.017 


\section{Abstract}

Objective: Selenium deficiency is a risk factor for Kashin-Beck disease (KBD), an endemic osteoarthropathy. Although promoter hypermethylation of glutathione peroxidase 3 (GPX3) (a selenoprotein) has been identified in several cancers, little is known about promoter methylation and expression of GPX3 and their relation to selenium in KBD. The present study was thus conducted to investigate this research question.

Methods: Methylation and expressions of GPX3 in whole blood drawn from 288 KBD patients and 362 healthy controls and in chondrocyte cell line were evaluated using methylation-specific PCR and qRT-PCR, respectively. The protein levels of PI3K/Akt/c-fos signaling in the whole blood and chondrocyte cell line were determined with Western blotting. Chondrocytes apoptosis were detected by Hoechst 33342 and Annexin V-FITC/PI staining.

Results: GPX3 methylation was increased, GPX3 mRNA was decreased, and protein levels in the PI3K/Akt/c-fos signaling pathway were up-regulated in the whole blood collected from KBD patients as compared with healthy controls. Similar results were obtained for chondrocytes injured by oxidative stress. There was a significant, decreasing trend in GPX3 expression across groups of unmethylation, partial methylation, and complete methylation for GPX3, in sequence. Compared with unmethylation group, protein levels in PI3K/Akt/c-fos pathway were enhanced in partial and complete methylation groups. Treatment of chondrocytes with sodium selenite resulted in reduced methylation and increased expression of $G P X 3$ as well as down-regulated level of PI3K/Akt/c-fos proteins.

Conclusions: The methylation and expression of GPX3 and expression of PI3K/Akt/c-fos pathway are altered in KBD and these changes are reversible by selenium supplementation. 
Key words: Kashin-Beck disease; glutathione peroxidase 3; DNA methylation; $\mathrm{PI} 3 \mathrm{~K} / \mathrm{Akt} / \mathrm{c}-$ fos signaling pathway; selenium 


\section{Introduction}

Kashin-Beck Disease (KBD) is an endemic osteoarthropathy that primarily occurs among children and adolescents and is manifested by joint pain, arthritis, muscle atrophy, deformed joints, and even dwarfism (1). The main pathological features of KBD are cartilage necrosis and excessive apoptosis of chondrocytes in the deep layers of epiphysis and articular cartilage $(2,3)$. KBD is prevalent in a large belt extending from Southeast Siberia, North Korea, Northeast China, to Southwest China (4). In China, more than 104 million people reside in KBD-affected areas, and 567,637 people were diagnosed with the disease, including 12,730 patients under 13 years of age (5). The etiology and pathogenesis of KBD largely remain unclear. A growing body of epidemiological evidence suggests that KBD develops as a consequence of the interaction between genes and environmental factors $(6,7)$. Selenium deficiency was observed to be an environmental risk factor for KBD in most epidemiological studies $(3,8)$, but the biochemical mechanisms by which selenium deficiency induces cartilage damage and thereby leads to an elevated risk of KBD are yet to be elucidated.

Epigenetics provides an optimal framework for investigating the mechanisms of environmental factors in KBD development. It is well documented that gene expression is regulated by epigenetic modifications, especially DNA methylation, in response to environmental exposures in a variety of diseases $(9,10)$. DNA hypermethylation is considered an important gene modification that prevents the recruitment of transcription factors and consequently suppresses the transcription of various genes (11). Several studies have revealed that selenium is involved in epigenetic modifications $(12,13)$. Selenoproteins are the main form of selenium that are responsible for numerous biological functions. One of these selenoproteins is glutathione peroxidases 3 (GPX3), a primary antioxidant enzyme in the human body (14). It is possible that epigenetic modifications of GPX3 induced by oxidative stress modulate antioxidant gene transcription and mRNA stability. GPX3 
differential methylation has been linked to cervical cancer, breast cancer, and multiple myeloma (15-17). Therefore, the methylation status of GPX3 may also play a role in the pathogenesis of KBD and other diseases related to oxidative stress.

Some studies have demonstrated that the phosphatidylinositol 3-kinase (PI3K)/protein kinase B (Akt)/c-fos signaling pathway is involved in celllular oxidative damage and apoptosis $(18,19)$. In addition, the differential level of PI3K/Akt pathway were found in KBD patients compared with controls and related to selenoprotein S (SEPS1) $-105 \mathrm{G}>\mathrm{A}$ polymorphisms (7). However, GPX3 DNA methylation status and its potential influence on cartilage damage among KBD patients have not been investigated up to date. Therefore, the present study was conducted to evaluate the association between GPX3 DNA methylation and $\mathrm{KBD}$ risk and to elucidate the mechanisms of GPX3 DNA methylation in the development of cartilage damage.

\section{Materials and methods}

\subsection{Study population}

KBD was diagnosed using the criteria of National Health Commission of the People's Republic of China (WS/T207-2010). A total of $288 \mathrm{KBD}$ cases and 362 controls were recruited from KBD endemic and non-endemic areas in Shaanxi province, respectively, which excluded osteoarthritis and other joint diseases. All subjects enrolled to the present study were Han Chinese. Cases and controls were comparable with regard to age and sex. The study protocol was approved by the Human Ethics Committee of Xi'an Jiaotong University, and a written informed consent was obtained from each of the study subjects prior to in-person interview and blood collection.

A peripheral venous blood sample $(5 \mathrm{ml})$ procured from each of study subjects were extracted for DNA, total mRNA, and proteins, and stored in freezers $\left(-80^{\circ} \mathrm{C}\right)$ until analysis. 
Independent experiments for GPX3 DNA methylation, GPX3 mRNA expression, and protein level described below were each repeated at least three times.

\subsection{GPX3 DNA methylation}

Genomic DNA were extracted from blood and chondrocytes using TIANamp Genomic DNA Kit (Tiangen Biotech, Beijing, China). Bisulfite conversion for methylation-specific polymerase chain reaction (MSP) was performed with EZ DNA Methylation Kit (ZYMO Research, Irvine, CA) according to the manufacturer's instructions. The methylation primers and unmethylation primers were used to amplify the 177-bp and 186-bp products from target sequences, respectively (20). The primer sequences are as follows: methylated primers (F: 5'-TATGTTATTGTCGTTTCGGGAC-3'; R: 5'-GTCCGTCTAAAATATCCGACG-3') and unmethylated primers (F: 5'-TTTATGTTATTGTTGTTTTGGGATG-3'; $\quad$ R: 5'-ATCCATCTAAAATATCCAACACTCC-3'). After bisulfite modification, a 50 $\mu \mathrm{L}$ PCR was carried out in $5 \mu \mathrm{L} 10 \times \mathrm{PCR}$ buffer, $4 \mu \mathrm{L}$ dNTP mixture, $1 \mu \mathrm{L}$ of each primer, $3 \mu \mathrm{L}$ bisulfite-modified DNA, $35.75 \mu \mathrm{L}$ ddH2O, and $0.25 \mu \mathrm{L}$ hot-start Taq-polymerase (Takara, Mountain View, CA). Thermal cycler conditions included an initial denaturation step of $95^{\circ} \mathrm{C}$ for 10 min, followed by a three-step PCR program composed of $94^{\circ} \mathrm{C}$ for $30 \mathrm{sec}, 59^{\circ} \mathrm{C}$ for $30 \mathrm{sec}$, and $72^{\circ} \mathrm{C}$ for $30 \mathrm{sec}$ for 40 cycles, and a final extension of $72^{\circ} \mathrm{C}$ for $10 \mathrm{~min}$. PCR products were identified by $2 \%$ agarose gels and visualized under the Light Transilluminator (UVP, Upland, CA).

\subsection{GPX3 mRNA expression}

Total RNA was isolated from the whole blood and chondrocyte samples using Trizol Kit (Life Technologies, Carlsbad, CA). cDNAs were synthesized with the RevertAidTM First Strand cDNA Synthesis Kit (MBI, Fermentas, Vilnius, Lithuania). Relative quantification of GPX3 gene expression was performed using an iQe5 quantitative real-time PCR Detection Systems (Bio-Rad, Philadelphia, PA) and with $\beta$-actin as a reference gene. The primers used 
for qRT-PCR were F: 5'-TTCACGACATCCGCTGGAA-3' and R: 5'-CATCTTGACGTTGCTGACCGT-3' for GPX3 as well as F: 5'-GAACGGTGAAGGTGACAGCAG-3' and R: 5'-GTGGACTTGGGAGAGGACT-3' for $\beta$-actin. Amplification was run in a $20 \mu \mathrm{L}$ reaction mixture containing $1.6 \mu \mathrm{L}$ of cDNA, 0.8 $\mu \mathrm{L}$ of each primer, $10 \mu \mathrm{L} 2 \times \mathrm{SYBR}$ Premix Ex Taq $\mathbb{I}$ (Takara, Mountain View, CA), and 6.8

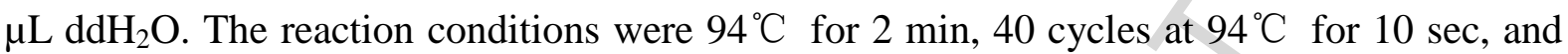
$60^{\circ} \mathrm{C}$ for $15 \mathrm{sec}$, with a final extension at $72^{\circ} \mathrm{C}$ for $30 \mathrm{sec}$. All reactions were performed in duplicate. The data were normalized to the expression level of $\beta$-actin and analyzed by iQe5 software (version 2.0, Bio-Rad).

\subsection{Protein level}

Total proteins were extracted from whole blood and chondrocytes using Trizol Kit (Life Technologies, Carlsbad, CA) according to the manufacturer's instructions. Samples were separated by $10 \%$ SDS-PAGE and transferred to NC membranes (Millipore Ireland BV, Cork, Ireland). The membranes were blocked with $5 \%$ non-fat-milk at $37^{\circ} \mathrm{C}$ for 1 hour. The primary antibodies against G $\beta \gamma$, PI3Kp110, pAkt, c-fos, total Akt and $\beta$-actin were diluted with a ratio of 1: 500 (all purchased from Sigma, Fremont, CA) and incubated overnight at $4^{\circ} \mathrm{C}$. After immunoreactive bands were detected with anti-rabbit $\operatorname{IgG}(1: 10,000$ dilution) (Pierce Company, WI), blots were developed using enhanced chemiluminescence (ECL) and visualized by gel imaging system (GDS-8000).

\subsection{Oxidative damage induced by $t B H P$ in $C 28 / I 2$ human chondrocytes}

The human C28/I2 chondrocytes (a human cell line provided by Dr. Mary Goldring from Cornell University) were cultured in DMEM/F-12 (Hyclone, Logan, UT) containing 12\% (v/v) fetal bovine serum (SiJiQing Bio-Technique Co. Ltd., Zhejiang, China) and 1\% (v/v) penicillin-streptomycin solution in a humidified incubator with $5 \% \mathrm{CO}_{2}$ at $37^{\circ} \mathrm{C}$. When the cells grew and reached $90 \%$ confluence in dishes, they were seeded on 96-well culture plates. 
The experiment was conducted in 6 groups: control group (C), purely Se group (S, 0.10 $\mu \mathrm{g} / \mathrm{mL}$ ), tert-butyl hydroperoxide (tBHP) injury group (O, tBHP $150 \mu \mathrm{mol} / \mathrm{L})$, low Se group (OS1, $\left.0.05 \mu \mathrm{g} / \mathrm{mL} \mathrm{Na}_{2} \mathrm{SeO}_{3}+150 \mu \mathrm{mol} / \mathrm{L} \mathrm{tBHP}\right)$, medium Se group (OS2, $0.10 \mu \mathrm{g} / \mathrm{mL}$ $\left.\mathrm{Na}_{2} \mathrm{SeO}_{3}+150 \mu \mathrm{mol} / \mathrm{L} \mathrm{tBHP}\right)$ and high Se group (OS3, $0.15 \mu \mathrm{g} / \mathrm{mL} \mathrm{Na}_{2} \mathrm{SeO}_{3}+150 \mu \mathrm{mol} / \mathrm{L}$ tBHP). OS1, OS2 and OS3 were treated with different concentrations of $\mathrm{Na}_{2} \mathrm{SeO}_{3}(0.05,0.10$ and $0.15 \mu \mathrm{g} / \mathrm{mL}$ ) for 24 hours, and then treated with $150 \mu \mathrm{mol} / \mathrm{L}$ tBHP for 24 hours.

\subsection{Chondrocyte apoptosis}

Chondrocytes were stained with 2\% Hoechst 33342 in DMEM/F-12 containing 12\% fetal bovine serum and then incubated at $37^{\circ} \mathrm{C}$ for $30 \mathrm{~min}$. Apoptotic chondrocytes with clear condensation and small bright nuclei were evaluated using a fluorescence microscope. The apoptotic rate of chondrocytes were measured using flow cytometry. Chondrocytes were collected and stained with Annexin V-FITC/PI according to the manufacturer's instructions (ROCHE, Basle, Switzerland). Cell fluorescence was detected by flow cytometry (BD, CA), and counts and percentages of live (annexin V-/PI-), early apoptotic (annexin V+/PI-), or late apoptotic/necrotic (annexin $\mathrm{V}+/ \mathrm{PI}+$ ) cells were obtained. The apoptotic rates were analyzed using Cell Quest software version 1.0 (BD, Franklin Lakes, NJ).

\subsection{Statistical analysis}

The Kolmogorov-Smirnov test was used to evaluate the normality of the distribution. Continuous variables were denoted by mean \pm standard deviation (SD), and differences in measured assays between two groups were assessed by two-tailed Student's t-test, as well as the differences among three or more groups were examined by one-way ANOVA. Categorical variables were presented as frequency counts, and differences in these variables were compared by Chi-square test. Odds ratio (OR) and $95 \%$ confidence interval (CI) were calculated to evaluate risk of KBD in relation to GPX3 methylation. A p-value of $<0.05$ 
(two-sided) was considered statistically significant. All statistical analyses were performed with the SPSS 23.0 software (SPSS Inc., Chicago, IL).

\section{Results}

\subsection{Demographic characteristics of study population}

The baseline characteristics of the study population are shown as follows. Mean age (SD) was 51.9 (3.4) years for $288 \mathrm{KBD}$ patients (151 men and 137 women) and 52.3 (3.8) years for 362 controls (168 men and 194 women). There were no significant differences in age ( $p=$ $0.16)$ and $\operatorname{sex}(p=0.13)$ composition between the patients and controls.

Furthermore, 50 cases and 50 controls were randomly selected from the above population for detection of GPX3 methylation and gene expression. The demographic characteristics of the samples were shown in Table 1. No statistically significant differences were observed between both groups in age $(\mathrm{P}=0.202)$ and $\operatorname{sex}(\mathrm{P}=0.841)$.

\subsection{GPX3 methylation in whole blood}

The GPX3 methylation status of peripheral blood mononuclear cells (PBMCs) procured from $50 \mathrm{KBD}$ patients and 50 healthy controls were examined using MSP. These patients and controls were randomly selected from all patients and controls enrolled to the present study. Complete methylation of GPX3 was identified in $16 \%$ of KBD patients, but only in $2 \%$ of controls $(p=0.036)$. Results showed that the GPX3 hypermethylation were associated with an increased risk of KBD [OR (95\% CI): 9.33, 95\% (1.12 77.7)].

\section{3. mRNA expression of GPX3 in KBD patients}

GPX3 mRNA level in whole blood was determined by qRT-PCR, and $\beta$-actin cDNA was the reference in the experiments. Results showed that GPX3 expression levels were significantly reduced in KBD patients compared with those of health individuals $(p=0.001)$ (Figure 1A). 


\subsection{Protein level of PI3K/Akt/c-fos signaling pathway in KBD patients}

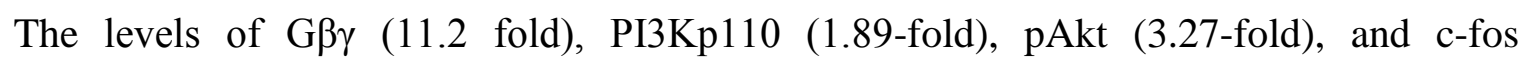
(16.5-fold) were significantly increased in KBD group compared with those of control group (all $p<0.001$ ) (Figure 2), suggesting that PI3K/Akt/c-fos signaling pathway was overexpressed in KBD patients.

\subsection{Alterations in oxidatively injured chondrocytes}

tBHP was used to induce the oxidatively damaged chondrocyte model. The effects of oxidative stress on cell apoptosis, methylation and mRNA level of GPX3, and protein levels of PI3K/Akt/c-fos were evaluated in human C28/I2 chondrocytes. Results with hoechst33342 staining showed that the number of chondrocyte apoptosis in tBHP injury group (65.96 \pm 3.98) was significantly increased compared with that of the control group $(1.30 \pm 0.32)$. However, the number of chondrocyte apoptosis was decreased in all three selenium supplement groups (OS1: $14.54 \pm 2.02$, OS2: $4.15 \pm 0.19$, OS3: $14.74 \pm 2.56$ ) compared with tBHP injury group $(p<0.05)$ (Figure 3 ). The late apoptotic rate of chondrocytes in $\mathrm{C}, \mathrm{S}, \mathrm{O}$, OS1, OS2, and OS3 groups were $0.09 \%, 7.05 \%, 41.01 \%, 39.47 \%, 36.75 \%$ and $29.36 \%$, respectively (Figure 4). The late apoptotic rate of chondrocyte in $\mathrm{O}$ group was increased compared with C group, but it was reduced in OS1, OS2, OS3 groups, suggesting that Se supplement at concentrations from $0.05 \mu \mathrm{g} / \mathrm{mL}$ to $0.15 \mu \mathrm{g} / \mathrm{mL}$ could decrease apoptotic rate in human C28/I2 chondrocytes and exert a dose-dependent trends.

Complete methylation and unmethylation of GPX3 was observed in the oxidative injury group and control group, respectively, indicating that oxidative damage resulted in methylation of this gene (Table 2). GPX3 expression was lower in the tBHP injury group than in the control group $(p<0.001)$ (Figure 1B). Protein levels of G $\beta \gamma$, PI3Kp110, pAkt and c-fos were detected in chondrocytes. As shown in Figure 5, the protein levels of G $\beta \gamma$, 
PI3Kp110, pAkt, and c-fos were increased in the tBHP group compared with those of the control group (all $p<0.0001$ ).

\subsection{GPX3 expression under different methylation status in chondrocytes}

The results revealed that there was a significant difference on mRNA levels of GPX3 among the three groups $(p<0.0001)$. The mRNA level of $G P X 3$ in unmethylation group was higher than that in partial methylation group and in complete methylation group (all $p<0.01$ ), while mRNA level of GPX3 was higher in the partial methylation group than in complete methylation group $(p<0.01)$. These results indicate that reduced GPX3 gene expression was mediated through by its increased methylation (Figure 1C).

3.7. Protein levels of PI3K/Akt/c-fos signaling in relation to methylation status in chondrocytes

Compared with unmethylation group, protein levels of G $\beta \gamma$, PI3Kp110, pAkt, and c-fos were increased in partial methylation group (all $p<0.01$ ) and complete methylation group (all $p<0.01$ ). The protein levels of $\mathrm{G} \beta \gamma$, PI3Kp110, and c-fos in partial methylation group were significantly higher than those in complete methylation group (all $p<0.01$ ) (Figure 6), suggesting that the down-regulation of PI3K/Akt/c-fos protein level was correlated with a decreased GPX3 methylation.

3.8. Effects of Selenium on expression and methylation of GPX3 and PI3K/Akt/c-fos signaling in chondrocytes

It was found that GPX3 was completely methylated in the tBHP injury group, partially methylated in low Se and medium Se groups, and unmethylated in high Se group (Table 2), suggesting that selenium supplementation reduced GPX3 methylation. The GPX3 mRNA expression was significantly up-regulated in Se supplementation group than in the tBHP injury group (all $p<0.01$ ) (Figure 1B). Our results revealed that down-regulated GPX3 
expression was related to GPX3 hypermethylation in chondrocytes and Se treatment could up-regulate the mRNA expression levels of GPX3.

Compared with the $\mathrm{O}$ group, a significant reduction in protein levels were found for PI3Kp110 in OS2 and OS3 groups, G $\beta \gamma$ in OS1 and OS2, pAkt in OS3 group, and c-fos in three Se supplementation groups (all $p<0.01$ ) (Figure 5). These results suggest that there are a significantly up-regulated protein levels of PI3K/Akt/c-fos when chondrocytes are damaged by oxidative stress and that selenium supplementation can down-regulate protein levels of $\mathrm{PI} 3 \mathrm{~K} / \mathrm{Akt} / \mathrm{c}-$ fos signaling.

\section{Discussion}

The characteristics of pathological changes in KBD are the necrosis and excessive apoptosis in articular and epiphyseal plate cartilages. Oxidative stress has been identified to contribute to the pathogenesis of KBD (3). The serum levels of thiobarbituric acid were higher and GPX levels were lower in KBD patients than in controls, suggesting that selenium deficiency may alter oxidative stress in cartilage tissues (2). Recent studies have revealed that oxidative stress induced epigenetic dysregulation $(10,21)$, and accumulation of excess cellular reactive oxygen species resulted in increased DNA mutations and subsequent carcinogenesis (22). An increasing body of evidence has demonstrated that GPX3 promoter hypermethylation are present in several cancers (21-26). In addition, an in vitro reduction in GPX3 expression was associated with an elevated generation of reactive oxygen species and an increased formation of DNA damage in complex diseases or abnormal health conditions such as cancer and metabolic syndrome $(10,23)$. To date, however, little is known about the patterns of $G P X 3$ promoter methylation in KBD.

In the present study, we investigated the status of GPX3 promoter methylation between KBD patients and healthy controls. It was found that the frequency of GPX3 DNA methylation was significantly higher in KBD patients than in controls. In addition, GPX3 
hypermethylation was associated with a more than 9-fold increased risk of KBD. Our study offers novel evidence supporting that GPX3 promoter hypermethylation might be partially involved in the pathogenesis and etiology of KBD. DNA methylation and tissue-specific gene expression are the most common epigenetic alterations. DNA methylation has been used as biomarkers for early diagnosis, progression, and prognosis of various cancers (22-24). Our results showed that the expression of GPX3 was significantly down-regulated in KBD patients compared with healthy controls, indicating that DNA methylation might suppresses the expression of GPX3. Sabrina et al. (10) reported that GPX activity depended on the transcription levels of its related genes, which were themselves subject to regulation by methylation of their promoter regions. Therefore, it is possible that GPX3 expression was silenced in KBD patients related to GPX3 hypermethylation, resulting in reduced GPX3 activity and subsequent compromised antioxidative and antiapoptotic functions.

Although GPX3 promoter hypermethylation induced by oxidative stress has been observed to down-regulate its expression, other mechanisms modulating GPX3 expression are not well understood. DNA methylation is involved not only in transcriptional regulation and DNA damage repair, but also in intracellular signal transduction (10). PI3K/Akt/c-fos is the predominant signaling pathway of chondrocyte proliferation, growth, and apoptosis (27, 28). Similarly, GPX3 methylation is related to cell apoptosis (29). Therefore, we attempted to explore the relationship between GPX3 methylation and PI3K/Akt/c-fos signaling pathway. Of note, we found that the protein levels of G $\beta \gamma$, PI3Kp110, pAkt and c-fos in KBD patients were significantly elevated in KBD patients, indicating that PI3K/Akt/c-fos signaling is substantially enhanced in KBD patients.

Several studies have demonstrated that the excessive apoptosis of chondrocytes and oxidative stress plays a critical role in the pathophysiology of $\operatorname{KBD}(30,31)$; however, the underlying mechanisms remain unclear. The present study explored the mechanisms of $G P X 3$ 
methylation in oxidatively damaged chondrocytes. We found that GPX3 was completely methylated in oxidative damage group, but was unmethylated in control group and purely Se group. Furthermore, downregulated GPX3 expression was observed in oxidative damage group. These results indicated that oxidative stress could result in epigenetic dysregulation in chondrocytes. We also investigated the mRNA levels of GPX3 and protein levels of PI3K/Akt/c-fos signaling molecules under different degrees of GPX3 DNA methylation. It was found that the mRNA levels of GPX3 was significantly decreased in the complete methylation group compared with unmethylation group. The protein levels of PI3K/Akt/c-fos were higher in complete methylation group than in unmethylation group. Taken together, our study suggests that GPX3 promoter methylation might be associated with the downregulation of GPX3 and that the PI3K/Akt/c-fos signaling pathways is up-regulated by hypermethylation of GPX3. Hypermethylation status was often accompanied by an increased apoptosis of chondrocytes. Of particular interest is that aforementioned findings were similar to those obtained from KBD patients. Our study provides additional evidence for a role of GPX3 hypermethylation in chondrocyte apoptosis. Potential mechanisms for this observation might be that chondrocyte apoptosis arises as a consequence of GPX3 inactivation mediated through its hypermethylation and elevated levels of proteins involved in PI3K/Akt/c-fos signaling pathway. Similar findings were reported in several studies of multiple cancers in which GPX3 expression was down-regulated by its promoter hypermethylation, leading to reduced GPX3 capacity against oxidative damage $(14,20,24,25,32)$.

Epigenetics is a biological process that is modulated by environmental factors, especially nutrition (10). Selenium is a micronutrient that serves as a co-factor of GPX, a primary antioxidant enzyme. As mentioned above, selenium deficiency is a main risk factor of KBD, but its effects on cartilage damage are not fully understood. Therefore, it is important to elucidate biological mechanisms by which selenium deficiency is implicated in the etiology 
and molecular pathogenesis of KBD. Inadequate selenium nutritional status has also been linked to a variety of human diseases, including cancer and cardiovascular disease $(33,34)$. Recent studies have demonstrated that selenium plays an important role in epigenetic transcriptional regulation. Selenium can modulate DNA and histones to activate methylation-silenced genes (13) and regulate inflammatory gene expression at the epigenetic level (12). As the methylation is a reversible process, removal of methylation could restore GPX3 gene function (22). Our study showed that selenite treatment resulted in promoter DNA demethylation and reexpression of GPX3 as well as downregulation of protein levels of PI3K/Akt/c-fos. All of these changes might promote anti-apoptosis. Our study demonstrates that methylation status of $G P X 3$ could be reversed by selenium supplementation. Specifically, selenium could activate methylation-silenced $G P X 3$ gene by modifying DNA methylation and regulating PI3K/Akt/c-fos signaling pathway in chondrocytes.

In conclusion, our results showed that GPX3 promoter hypermethylation and down-regulated expression of GPX3 gene in KBD patients. Furthermore, GPX3 hypermethylation down-regulates its expression and up-regulates the proteins in the PI3K/Akt/c-fos signaling pathway. Selenium significantly protected against tBHP-induced chondrocyte apoptosis, possibly mediated through reduced GPX3 DNA methylation, increased GPX3 gene expression, and decreased PI3K/Akt/c-fos signaling. The findings of the present study will provide a new insight into the pathogenesis, etiology, and prevention of KBD. 


\section{Acknowledgements}

The authors thank all the volunteers who participant in this study. This study was supported by grants from National Natural Science Foundation of China (No. 81573104, 81773372,81172610 and 81673117).

\section{Conflict of interests}

The authors declare no conflict of interest.

\section{Authors' contributions}

All authors were involved in drafting the manuscript or critically revising it for important intellectual content and approved the final version of submitted manuscript. Yongmin Xiong takes final responsibility for the integrity of the work. Study conception and design: Lixin Han, Xiaoli Yang, Yongmin Xiong. Acquisition of data: Lixin Han, Xiaoli Yang, Zhaofang Li, Hao Ren, Baorong Li, Rongqiang Zhang, Dandan Zhang, Ziyun Shi, Jifeng Liu, Junling Cao. Analysis and interpretation of data: Yongmin Xiong, Lixin Han, Xiaoli Yang, Hao Ren, Jianjun Zhang, Wenyan Sun. 


\section{References}

1. Yamamuro T. Kashin-Beck disease: a historical overview. Int Orthop 2001;25:134-7.

2. Chen JH, Xue S, Li S, Wang ZL, Yang H, Wang W, et al. Oxidant damage in Kashin-Beck disease and a rat Kashin-Beck disease model by employing T-2 toxin treatment under selenium deficient conditions. $\mathbf{J}$ Orthop Res 2012;30:1229-37.

3. Guo X, Ma WJ, Zhang F, Ren FL, Qu CJ, Lammi MJ. Recent advances in the research of an endemic osteochondropathy in China: Kashin-Beck disease. Osteoarthritis Cartilage 2014;22:1774-83.

4. Luo R, Liu G, Liu W, Pei F, Zhou Z, Li J, et al. Efficacy of celecoxib, meloxicam and paracetamol in elderly Kashin-Beck disease (KBD) patients. Int Orthop 2011;35:1409-14.

5. Commission NHAF. China's Health And Family Planning Statistical Yearbook 2016. Beijing: Peking Union Medical College Press; 2016.

6. Sun LY, Li Q, Meng FG, Fu Y, Zhao ZJ, Wang LH. T-2 toxin contamination in grains and selenium concentration in drinking water and grains in Kaschin-Beck disease endemic areas of Qinghai Province. Biol Trace Elem Res 2012;150:371-5.

7. Du XA, Wang HM, Dai XX, Kou Y, Wu RP, Chen Q, et al. Role of selenoprotein S (SEPS1) -105G\&gt;A polymorphisms and PI3K/Akt signaling pathway in Kashin-Beck disease. Osteoarthritis Cartilage 2015;23:210-6.

8. Mo DX, Ding DX, Wang ZL, Zhang JJ, Bai C. Twenty-Year Research on Selenium Ralated to Kashin-Back Disease. Journal of Xi'an Medical University 1997:79-89.

9. Kim GH, Ryan JJ, Archer SL. The role of redox signaling in epigenetics and cardiovascular disease. Antioxid Redox Signal 2013;18:1920-36. 
10. Yara S, Lavoie JC, Levy E. Oxidative stress and DNA methylation regulation in the metabolic syndrome. Epigenomics 2015;7:283-300.

11. Rentsendorj O, Zhang X, Williams MC, Buehler PW, D'Agnillo F. Transcriptional Suppression of Renal Antioxidant Enzyme Systems in Guinea Pigs Exposed to Polymerized Cell-Free Hemoglobin. Toxics $2016 ; 4$.

12. Narayan V, Ravindra KC, Liao C, Kaushal N, Carlson BA, Prabhu KS. Epigenetic regulation of inflammatory gene expression in macrophages by selenium. J Nutr Biochem 2015;26:138-45.

13. Speckmann B, Grune T. Epigenetic effects of selenium and their implications for health. Epigenetics 2015;10:179-90.

14. Cohen S, Mehrabi S, Yao X, Millingen S, Aikhionbare FO. Reactive Oxygen Species and Serous Epithelial Ovarian Adenocarcinoma. Cancer Res J (N Y N Y) 2016;4:106-114.

15. Zhang X, Zheng Z, Yingji S, Kim H, Jin R, Renshu L, et al. Downregulation of glutathione peroxidase 3 is associated with lymph node metastasis and prognosis in cervical cancer. Oncol Rep 2014;31:2587-92.

16. Mohamed MM, Sabet S, Peng DF, Nouh MA, El-Shinawi M, El-Rifai W. Promoter hypermethylation and suppression of glutathione peroxidase 3 are associated with inflammatory breast carcinogenesis. Oxid Med Cell Longev 2014;2014:787195.

17. Kaiser MF, Johnson DC, Wu P, Walker BA, Brioli A, Mirabella F, et al. Global methylation analysis identifies prognostically important epigenetically inactivated tumor suppressor genes in multiple myeloma. Blood 2013;122:219-26.

18. Zha X, Wu G, Zhao X, Zhou L, Zhang H, Li J, et al. PRDX6 Protects ARPE-19 Cells from Oxidative Damage via PI3K/AKT Signaling. Cell Physiol Biochem 2015;36:2217-28. 
19. Zhang X, Zhang L, Yang H, Huang X, Otu H, Libermann TA, et al. c-Fos as a proapoptotic agent in TRAIL-induced apoptosis in prostate cancer cells. Cancer Res 2007;67:9425-34.

20. He Y, Wang Y, Li P, Zhu S, Wang J, Zhang S. Identification of GPX3 epigenetically silenced by CpG methylation in human esophageal squamous cell carcinoma. Dig Dis Sci 2011;56:681-8.

21. Zhou JD, Lin J, Zhang TJ, Ma JC, Yang L, Wen XM, et al. GPX3 methylation in bone marrow predicts adverse prognosis and leukemia transformation in myelodysplastic syndrome. Cancer Med 2017;6:267-274.

22. Liu Q, Jin J, Ying J, Sun M, Cui Y, Zhang L, et al. Frequent epigenetic suppression of tumor suppressor gene glutathione peroxidase 3 by promoter hypermethylation and its clinical implication in clear cell renal cell carcinoma. Int J Mol Sci 2015;16:10636-49.

23. Zhou JD, Yao DM, Zhang YY, Ma JC, Wen XM, Yang J, et al. GPX3 hypermethylation serves as an independent prognostic biomarker in non-M3 acute myeloid leukemia. Am J Cancer Res 2015;5:1786-94.

24. Pelosof L, Yerram S, Armstrong T, Chu N, Danilova L, Yanagisawa B, et al. GPX3 promoter methylation predicts platinum sensitivity in colorectal cancer. Epigenetics 2017;12:540-550.

25. Peng DF, Hu TL, Schneider BG, Chen Z, Xu ZK, El-Rifai W. Silencing of glutathione peroxidase 3 through DNA hypermethylation is associated with lymph node metastasis in gastric carcinomas. PLoS One 2012;7:e46214.

26. Cao S, Yan B, Lu Y, Zhang G, Li J, Zhai W, et al. Methylation of promoter and expression silencing of GPX3 gene in hepatocellular carcinoma tissue. Clin Res Hepatol Gastroenterol 2015;39:198-204.

27. Coskun D, Obakan P, Arisan ED, Coker-Gurkan A, Palavan-Unsal N. Epibrassinolide alters PI3K/MAPK signaling axis via activating Foxo3a-induced mitochondria-mediated apoptosis in colon cancer cells. Exp Cell Res 2015;338:10-21. 
28. Li X, LuValle P. Activating transcription factor 2 targets c-Fos, but not c-Jun, in growth plate chondrocytes. J Cell Biochem 2011;112:211-6.

29. Qu Y, Dang S, Hou P. Gene methylation in gastric cancer. Clin Chim Acta 2013;424:53-65.

30. Zhang BD, Guo X, Bai GL, Ping ZG, Zuo H, Ren FL, et al. The changes of nitric oxide, NO synthase and sFas/APO-1 in serum among the patients with Kashin-Beck disease. Chinese Journal of Endemiology 2004:79-82.

31. Wang W, Wei S, Luo M, Yu B, Cao J, Yang Z, et al. Oxidative stress and status of antioxidant enzymes in children with Kashin-Beck disease. Osteoarthritis Cartilage 2013;21:1781-9.

32. Chen B, Rao X, House MG, Nephew KP, Cullen KJ, Guo Z. GPx3 promoter hypermethylation is a frequent event in human cancer and is associated with tumorigenesis and chemotherapy response. Cancer Lett 2011;309:37-45.

33. Qiao B, He B, Cai J, King-Yin LA, He W. Induction of oxidative stress and cell apoptosis by selenium: the cure against oral carcinoma. Oncotarget 2017;8:113614-113621.

34. Gharipour M, Sadeghi M, Behmanesh M, Salehi M, Nezafati P, Gharpour A. Selenium Homeostasis and Clustering of Cardiovascular Risk Factors: A Systematic Review. Acta Biomed 2017;88:263-270. 


\section{Figure legends}

Figure 1. The GPX3 expression in KBD patients and chondrocytes. (A) The expression of GPX3 in whole blood among KBD patients and controls. The mRNA levels of GPX3 were significantly down-regulated in KBD group as compared with control group ( $p=0.001)$. (B) The GPX3 expression in cultured human C28/I2 chondrocyte. The expression levels of GPX3 were lower in the $\mathrm{O}$ group than in the $\mathrm{C}$ group $(p<0.001)$. Compared with the $\mathrm{O}$ group, the GPX3 expressions in three Se supplement group were up-regulated significantly $(p<0.05)$. C: control group. S: purely Se group $\left(0.05 \mu \mathrm{g} / \mathrm{mL} \mathrm{Na}_{2} \mathrm{SeO}_{3}\right)$. O: tBHP injury group (tBHP 150 $\mu \mathrm{mol} / \mathrm{L})$. OS1: low Se group $\left(0.05 \mu \mathrm{g} / \mathrm{mL} \mathrm{Na}_{2} \mathrm{SeO}_{3}+150 \mu \mathrm{mol} / \mathrm{L}\right.$ tBHP $)$. OS2: medium Se group $\left(0.1 \mu \mathrm{g} / \mathrm{mL} \mathrm{Na}_{2} \mathrm{SeO}_{3}+150 \mu \mathrm{mol} / \mathrm{L}\right.$ tBHP $)$. OS3: high Se group $\left(0.15 \mu \mathrm{g} / \mathrm{mL} \mathrm{Na}_{2} \mathrm{SeO}_{3}\right.$ $+150 \mu \mathrm{mol} / \mathrm{L}$ tBHP). (C) The GPX3 expressions in chondrocyte under different methylation status. The mRNA level of GPX3 in unmethylation group was higher than that in partial methylation group and in complete methylation group (all $p<0.01$ ), while mRNA level of GPX3 was higher in the partial methylation group than in complete methylation group $(p<$ 0.01). UM: unmethylation CM: complete methylation. PM: partial methylation.

* represents significance at $p<0.05$.

Figure 2. Protein levels of PI3K/Akt/c-fos in whole blood between the KBD patients and controls. (A) The protein level of G $\beta \gamma$. (B) The protein level of PI3Kp110. (C) The protein level of pAkt. (D) The protein level of c-fos. All protein levels in KBD group were significantly higher than that in control group $(p<0.001) . \mathrm{K} 1, \mathrm{~K} 2$ and $\mathrm{K} 3$ : samples from KBD patients; C1, C2 and C3: samples from control subjects. *represents significance at $p<$ 0.05 . 
Figure 3. The apoptosis numbers were detected by Hoechst 33342 staining in human C28/I2 chondrocyte. Compared with $\mathrm{C}$ and $\mathrm{S}$ groups, the apoptosis numbers were obviously increased in $\mathrm{O}$ group, while the apoptosis numbers were reduced by selenite treatment. C: Control group. S: purely Se group $\left(0.05 \mu \mathrm{g} / \mathrm{mL} \mathrm{Na}_{2} \mathrm{SeO}_{3}\right)$. O: tBHP injury group (tBHP 150 $\mu \mathrm{mol} / \mathrm{L})$. OS1: low Se group $\left(0.05 \mu \mathrm{g} / \mathrm{mL} \mathrm{Na}_{2} \mathrm{SeO}_{3}+150 \mu \mathrm{mol} / \mathrm{L} \mathrm{tBHP}\right) . \mathrm{OS} 2:$ medium Se group $\left(0.1 \mu \mathrm{g} / \mathrm{mL} \mathrm{Na}_{2} \mathrm{SeO}_{3}+150 \mu \mathrm{mol} / \mathrm{L}\right.$ tBHP $)$. OS3: high Se group $\left(0.15 \mu \mathrm{g} / \mathrm{mL} \mathrm{Na}_{2} \mathrm{SeO}_{3}\right.$ $+150 \mu \mathrm{mol} / \mathrm{L}$ tBHP).

Figure 4. The apoptosis rate were detected by Annexin V-FITC/PI double staining in cultured human C28/I2 chondrocyte. (A) Annexin V-FITC/PI staning in chondrocytes. (B) The late apoptosis rate in chondrocytes. The late apoptotis rate of chondrocyte in $\mathrm{O}$ group was increased compared with $\mathrm{C}$ group, but it was reduced in OS1, OS2 and OS3 groups. Numbers in each quadrant indicated the percentage of chondrocytes labeled with annexin V-FITC (top left), PI (bottom right), annexin V-FITC and PI (top right), and untaged (bottom left). C: Control group. S: purely Se group $\left(0.05 \mu \mathrm{g} / \mathrm{mL} \mathrm{Na}_{2} \mathrm{SeO}_{3}\right)$. O: tBHP injury group (tBHP $150 \mu \mathrm{mol} / \mathrm{L})$. OS1: low Se group $\left(0.05 \mu \mathrm{g} / \mathrm{mL} \mathrm{Na}_{2} \mathrm{SeO}_{3}+150 \mu \mathrm{mol} / \mathrm{L} \mathrm{tBHP}\right)$. OS2: medium Se group $\left(0.1 \mu \mathrm{g} / \mathrm{mL} \mathrm{Na}_{2} \mathrm{SeO}_{3}+150 \mu \mathrm{mol} / \mathrm{L}\right.$ tBHP $)$. OS3: high Se group $(0.15$ $\left.\mu \mathrm{g} / \mathrm{mL} \mathrm{Na}_{2} \mathrm{SeO}_{3}+150 \mu \mathrm{mol} / \mathrm{L} \mathrm{tBHP}\right)$.

Figure 5. Protein levels of PI3K/Akt/c-fos signaling in human C28/I2 chondrocytes. (A) The level of G $\beta \gamma$. (B) The protein level of PI3Kp110. (C) The protein level of pAkt. (D) The protein levels of c-fos. Compared with the $\mathrm{C}$ group, the protein level of G $\beta \gamma$, PI3Kp110, pAkt and c-fos protein were increased in the $\mathrm{O}$ group than those of the $\mathrm{C}$ group (all $p<0.0001$ ). Compared with the $\mathrm{O}$ group, a significant reduction in protein levels were found for PI3Kp110 in OS2 and OS3 groups, c-fos in three Se supplementation groups, G $\beta \gamma$ in OS1 
and OS2 (all $p<0.01)$, and pAkt OS3 groups $(p<0.01)$. C: control group. S: purely Se group $\left(0.05 \mu \mathrm{g} / \mathrm{mL} \mathrm{Na}_{2} \mathrm{SeO}_{3}\right)$. O: tBHP injury group (tBHP $\left.150 \mu \mathrm{mol} / \mathrm{L}\right)$. OS1: low Se group (0.05 $\left.\mu \mathrm{g} / \mathrm{mL} \mathrm{Na}_{2} \mathrm{SeO}_{3}+150 \mu \mathrm{mol} / \mathrm{L} \mathrm{tBHP}\right)$. OS2: medium Se group $\left(0.1 \mu \mathrm{g} / \mathrm{mL} \mathrm{Na}_{2} \mathrm{SeO}_{3}+150\right.$ $\mu \mathrm{mol} / \mathrm{L}$ tBHP). OS3: high Se group $\left(0.15 \mu \mathrm{g} / \mathrm{mL} \mathrm{Na}_{2} \mathrm{SeO}_{3}+150 \mu \mathrm{mol} / \mathrm{L} \mathrm{tBHP}\right) .{ }^{*}$ represents significance at $p<0.05$.

Figure 6. The protein levels of PI3K/Akt/c-fos signaling under different methylation status of GPX3 in chondrocytes. (A) The protein level of G $\beta \gamma$ with GPX3 methylation status. (B) The protein level of PI3Kp110 with GPX3 methylation status. (C) The protein level of pAkt with GPX3 methylation status. (D) The protein level of c-fos with GPX3 methylation status. Compared with unmethylation group, protein levels of G $\beta \gamma$, PI3Kp110, pAkt, and c-fos were increased in partial methylation group (all $p<0.01$ ) and complete methylation group (all $p<$ 0.01). The protein levels of $\mathrm{G} \beta \gamma, \mathrm{PI} 3 \mathrm{Kp} 110$, and $\mathrm{c}$-fos in partial methylation group were significantly higher than those in complete methylation group (all $p<0.01$ ). UM: unmethylation. CM: complete methylation. PM: partial methylation.

* represents significance at $p<0.05$. 
Table 1 Demographic characteristics of the study population

\begin{tabular}{ccccc}
\hline Characteristics & Cases $(\mathrm{n}=50)$ & Control $(\mathrm{n}=50)$ & Values & $P$ \\
\hline Sex, male/female & $26 / 24$ & $27 / 23$ & 0.04 & 0.841 \\
Age, mean \pm SD & $51.90 \pm 3.39$ & $52.85 \pm 3.98$ & 1.285 & 0.202 \\
\hline
\end{tabular}

${ }^{a}$ The difference of age between KBD and controls was performed using t-test and no statistical significance.

$\mathrm{b}$ The frequency of sex between KBD and controls was performed using $x^{2}$-test and no statistical significance. 
Table 2. GPX3 gene methylation in chondrocytes

GPX3 methylation status

\begin{tabular}{ccccc}
\cline { 2 - 3 } Groups & Complete & Partial methylation & Unmethylation & \\
& Methylation & $\mathrm{n}(\%)$ & $\mathrm{n}(\%)$ & \\
$\mathrm{n}(\%)$ & 0 & $24(100.00)$ & \\
$\mathrm{C}$ & 0 & 0 & $24(100.00)$ & \\
O & $23(95.83)$ & $1(4.17)$ & 0 & $<0.001^{\mathrm{a}}$ \\
OS1 & $3(13.04)$ & $20(86.96)$ & 0 & $<0.001^{\mathrm{b}}$ \\
OS2 & $2(8.70)$ & $21(91.30)$ & 0 & $<0.001^{\mathrm{b}}$ \\
OS3 & 0 & $1(4.35)$ & $22(95.65)$ & $<0.001^{\mathrm{b}}$ \\
\hline
\end{tabular}

C: control group; $\mathrm{S}$ : purely selenium group $\left(0.05 \mu \mathrm{g} / \mathrm{mL} \mathrm{Na}_{2} \mathrm{SeO}_{3}\right) ; \mathrm{O}$ : tBHP injury group (tBHP $150 \mu \mathrm{mol} / \mathrm{L})$; OS1: low selenium group $\left(0.05 \mu \mathrm{g} / \mathrm{mL} \mathrm{Na}_{2} \mathrm{SeO}_{3}+150 \mu \mathrm{mol} / \mathrm{L} \mathrm{tBHP}\right)$; OS2: medium selenium group $\left(0.1 \mu \mathrm{g} / \mathrm{mL} \mathrm{Na}_{2} \mathrm{SeO}_{3}+150 \mu \mathrm{mol} / \mathrm{L}\right.$ tBHP $)$; OS3: high selenium group $\left(0.15 \mu \mathrm{g} / \mathrm{mL} \mathrm{Na}_{2} \mathrm{SeO}_{3}+150 \mu \mathrm{mol} / \mathrm{L}\right.$ tBHP $)$.

a p-value for comparing $\mathrm{O}$ group with $\mathrm{C}$ groups, b p-value for comparing $\mathrm{O}$ group with OS1, OS2, OS3. 


\section{Hightlights:}

1. GPX3 DNA hypermethylation and low expression of gene existed in KBD patients.

2. Up-regulation of PI3K/Akt/c-fos in KBD patients and oxidative damage chondrocytes.

3. Hypermethylation and low gene expression of GPX3 found in apoptotic chondrocyte.

4. GPX3 hypermethylation related to changes in gene expression and PI3K/Akt/c-fos.

5. Methylation, gene expression of GPX3 and PI3K/Akt/c-fos improved by Se supplement. 

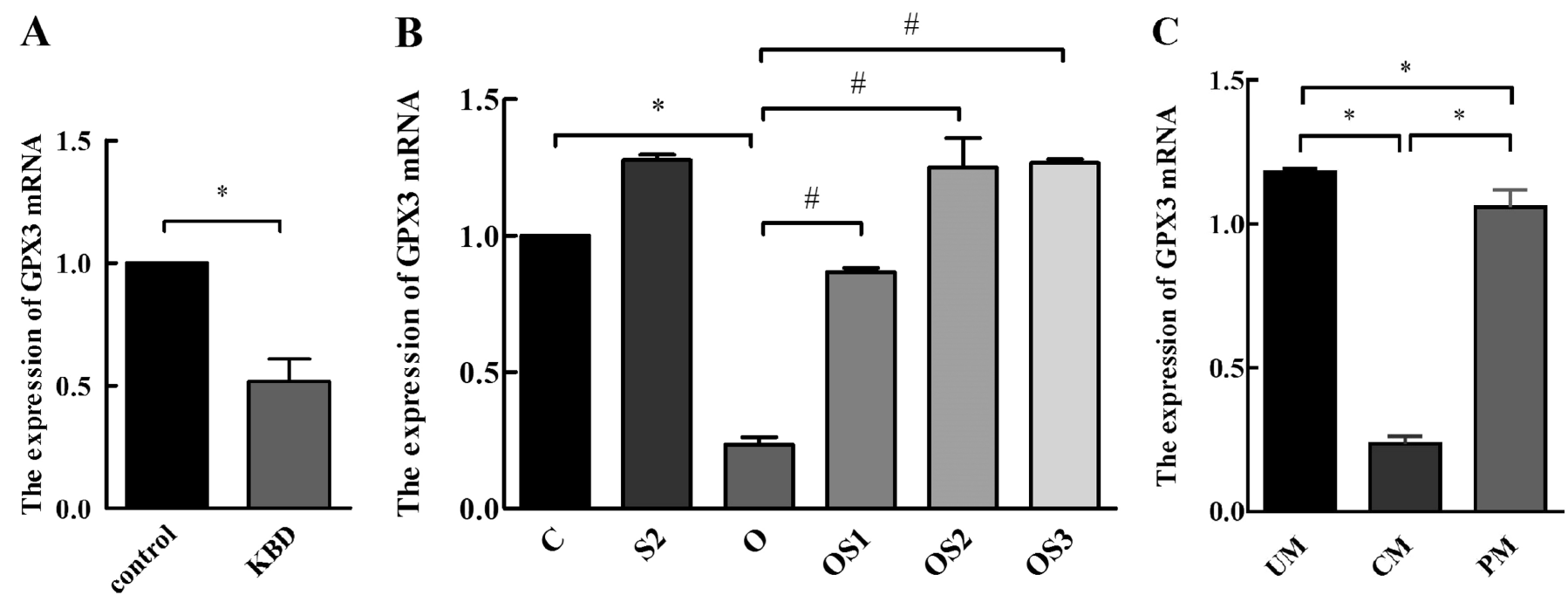
A

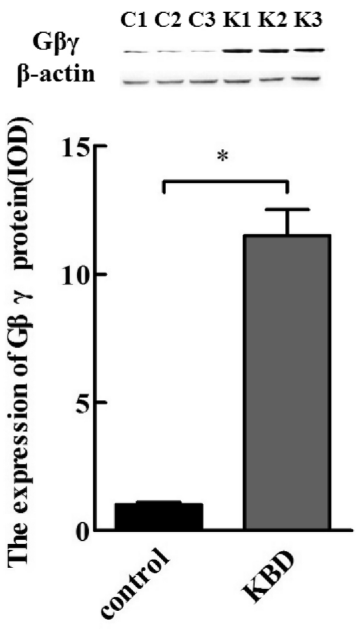

C

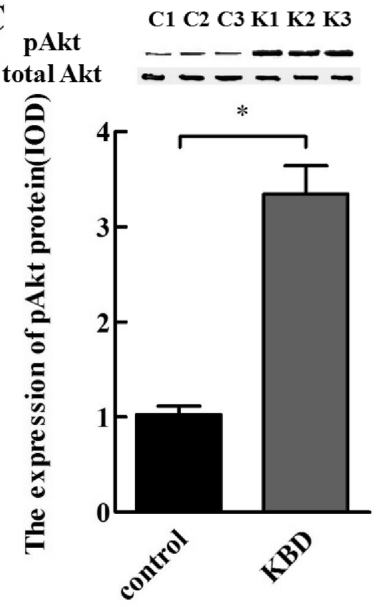

B
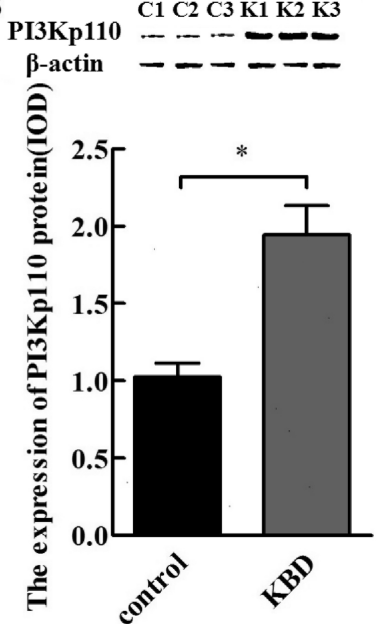

D
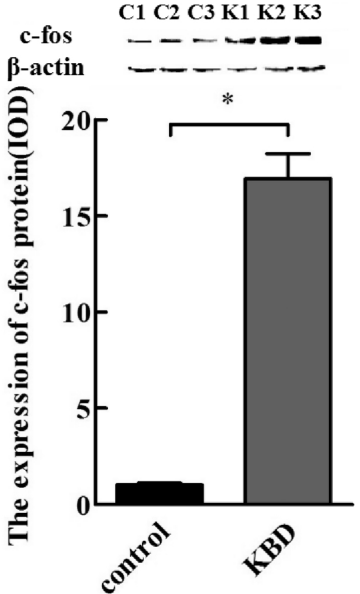


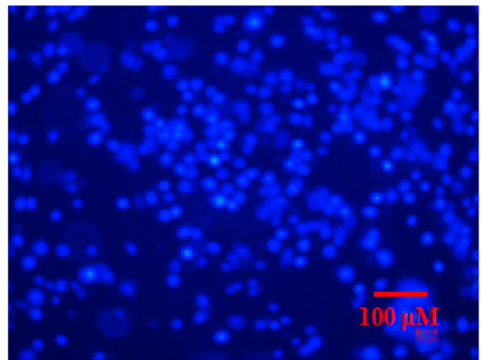

C group

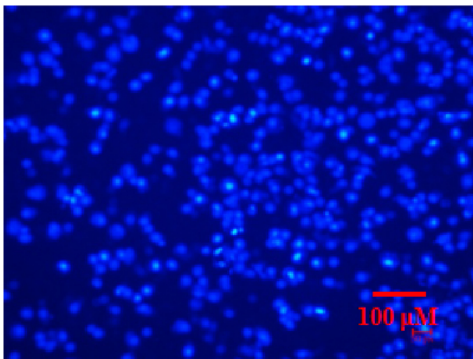

OS1 group

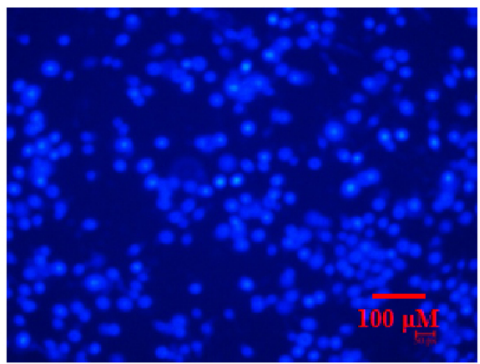

S2 group

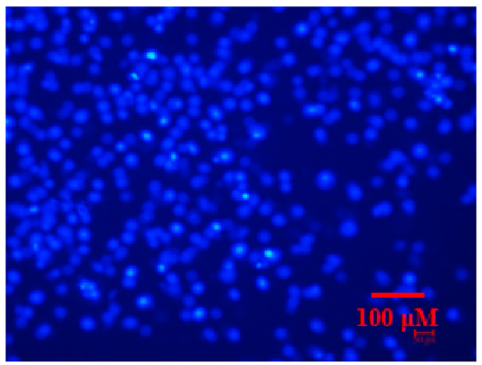

OS2 group

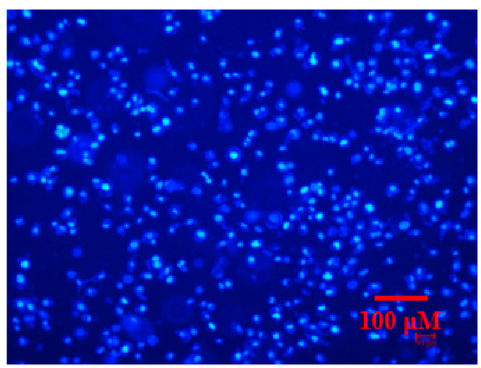

O group

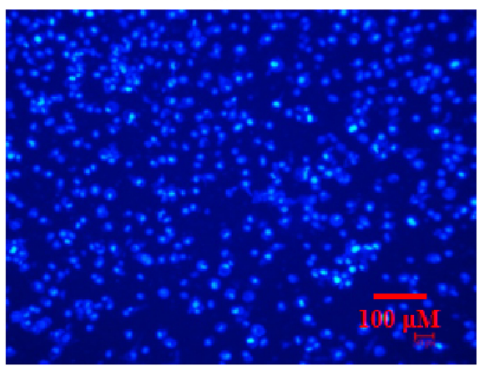

OS3 group

Figure 3 


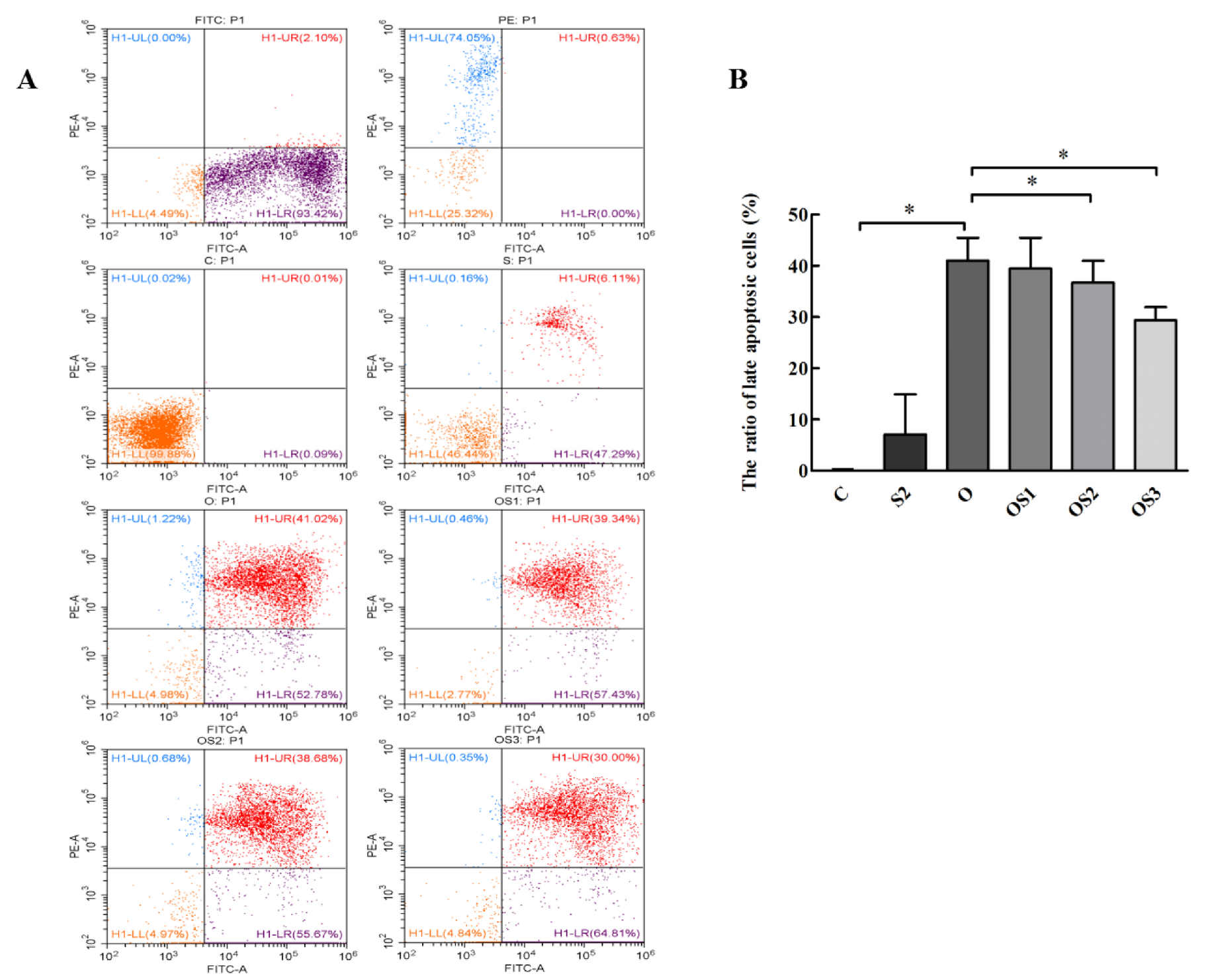

Figure 4 


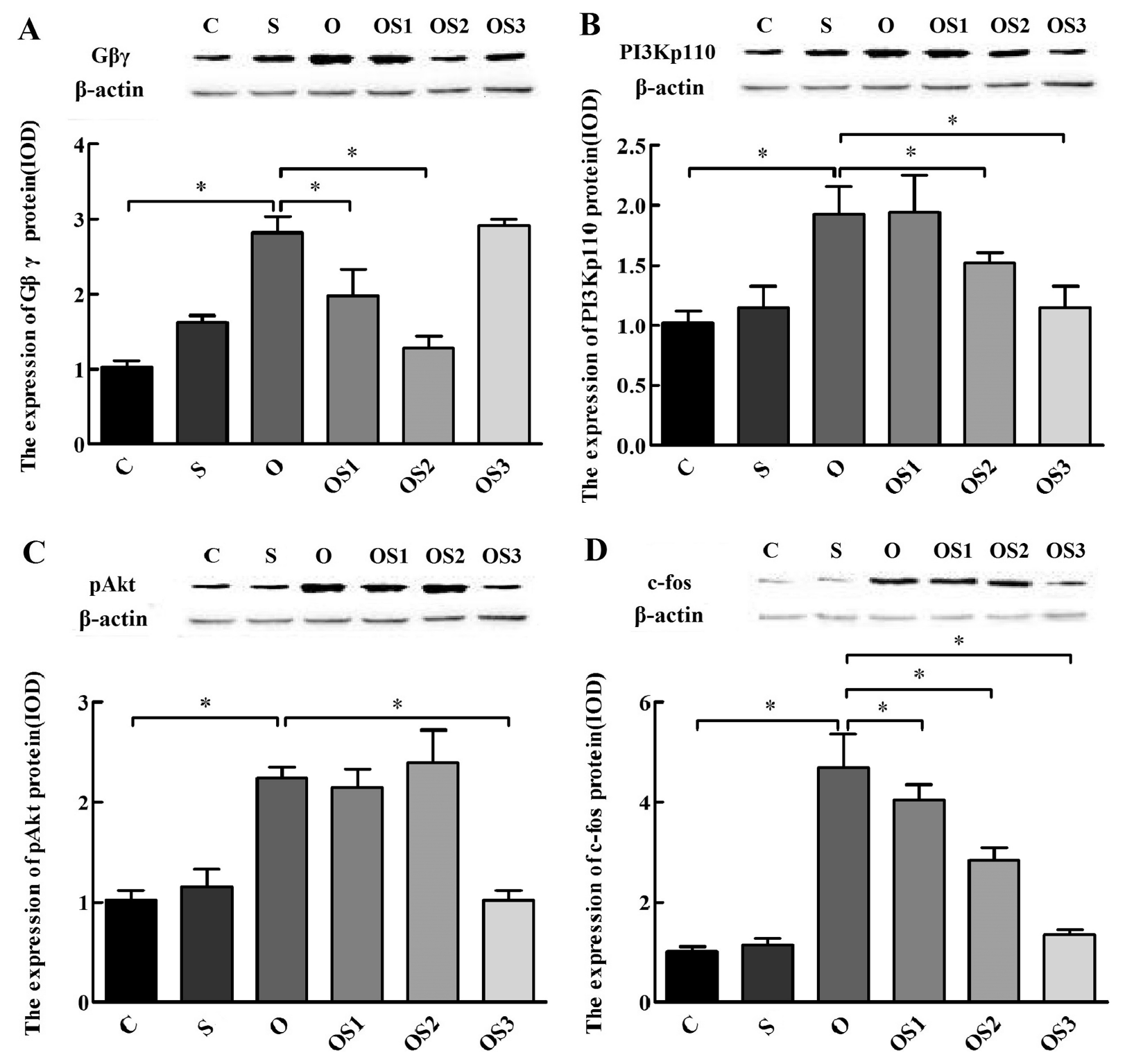

Figure 5 


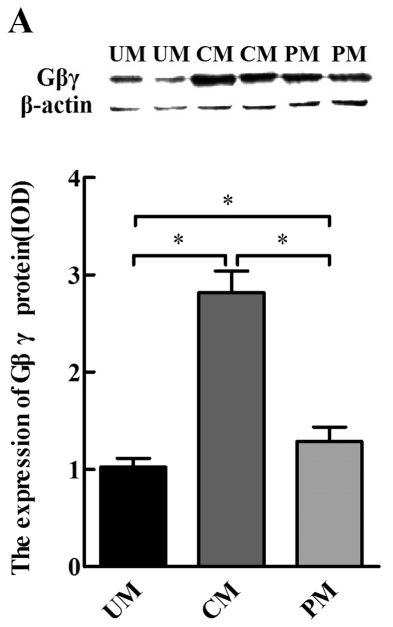

C

UMUM CM CM PM PM pAkt $=-2=$ $\beta$-actin - - - -

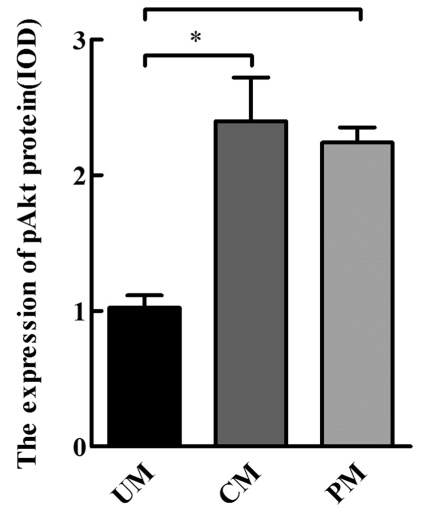

Figure 6 